7th International Workshop on Astronomy and

Relativistic Astrophysics (IWARA 2016)

International Journal of Modern Physics: Conference Series

Vol. 45 (2017) 1760066 (5 pages)

(C) The Author(s)

DOI: $10.1142 /$ S2010194517600667

\title{
Exploring the BEC-BCS Crossover in a Cold and Magnetized 2-Color QCD
}

\author{
Dyana C. Duarte \\ Departamento de Física, Universidade Federal de Santa Maria (UFSM) \\ 97105-900 Santa Maria, RS, Brazil \\ dyana.c.duarte@gmail.com \\ Pablo G. Allen \\ Departamento de Física Teórica, Comisión Nacional de Energía Atómica (CNEA) \\ Av. Libertador 8250, 1429 Buenos Aires, Argentina \\ pabloallen1@gmail.com \\ Ricardo L. S. Farias \\ Departamento de Fúsica, Universidade Federal de Santa Maria (UFSM) \\ 97105-900 Santa Maria, RS, Brazil \\ Department of Physics, Kent State University (KSU) \\ Kent, OH 44242, USA \\ ricardo.farias@ufsm.br \\ Patrícia H. A. Manso \\ Departamento de Física Teórica, Universidade do Estado do Rio de Janeiro (UERJ) \\ 20550-013 Rio de Janeiro, RJ, Brazil \\ Centro Federal de Educação Tecnológica Celso Suckow da Fonseca \\ Campus Maria da Graça, Rua Miguel Ângelo 96 \\ 20785-223 Rio de Janeiro, RJ, Brazil \\ phmansophy@gmail.com \\ Norberto N. Scoccola \\ Departamento de Física Teórica, Comisión Nacional de Energía Atómica (CNEA) \\ Av. Libertador 8250, 1429 Buenos Aires, Argentina \\ Departamento de Física Teórica, Comisión Nacional de Energía Atómica (CONICET) \\ Rivadavia 1917, 1033 Buenos Aires, Argentina \\ Universidad Favaloro \\ Solís 453, 1078 Buenos Aires, Argentina \\ scoccola@tandar.cnea.gov.ar
}

Published 15 August 2017

This is an Open Access article published by World Scientific Publishing Company. It is distributed under the terms of the Creative Commons Attribution 4.0 (CC-BY) License. Further distribution of this work is permitted, provided the original work is properly cited. 


\begin{abstract}
We study the BEC-BCS crossover in the presence of an external magnetic field for a two color NJL model with diquark interactions, giving special attention to the regularization scheme. We found a inverse magnetic catalysis on the critical chemical potentials, both for BEC phase transition and the BEC-BCS crossover for small values of magnetic fields, and a magnetic catalysis for large $e B$.
\end{abstract}

Keywords: BEC-BCS Crossover, Magnetic Fields, Regularization Scheme.

PACS numbers: 24.10.Jv, 25.75.Nq

\title{
1. Introduction
}

There are many motivations to study the phase structure of quantum chromodynamics (QCD), related to investigations of the relativistic heavy ion collisions, compact stars and the early universe. Moreover, the QCD phase diagram remains poorly understood due to the well known sign problem ${ }^{1}$, despite the many efforts dedicated to its description in recent years. Further motivated by the fact that strong magnetic fields may be produced in noncentral heavy-ion collisions, investigations of the effects produced by a magnetic field in the phase diagram of strongly interacting matter became a subject of great interest in recent years ${ }^{2}$. It is generally expected that there should exist a crossover from Bose-Einstein condensation (BEC) to Bardeen-Cooper-Shriffer condensation (BCS) for diquarks at finite baryon density. This crossover can be observed in different ways, such as increasing the coupling constant of the attractive interactions or changing the charge number through the variation of the chemical potential ${ }^{3}$. In this work we study the BEC-BCS crossover for a NJL model with diquark interactions in the presence of an external magnetic field, giving particular attention to different regularization schemes used in the literature ${ }^{4}$. A thorough comparison of results is performed for the case of a cold and magnetized two-color NJL model.

\section{2. $N_{c}=2$ NJL Model in an External Magnetic Field}

The Lagrangian density of a two-color and two-flavor NJL-type model in the presence of an external electromagnetic field, is given by

$$
\mathcal{L}=\bar{\psi}\left(i \not D-m_{c}\right) \psi+G_{S}\left[(\bar{\psi} \psi)^{2}+\left(\bar{\psi} i \gamma_{5} \tau \psi\right)^{2}\right]+G_{D}\left(\bar{\psi} i \gamma_{5} \tau_{2} t_{2} C \bar{\psi}^{T}\right)\left(\psi^{T} C i \gamma_{5} \tau_{2} t_{2} \psi\right)
$$

where $m_{c}$ is the current fermion mass, and $G_{S}=G_{D}=G$ (see Ref. ${ }^{5}$ ). The coupling of quarks to electromagnetic field $\mathcal{A}_{\mu}$ is given by $D_{\mu}=\partial_{\mu}-i Q \mathcal{A}_{\mu}$, where $Q$ is the usual quark charge matrix $Q=\operatorname{diag}\left(q_{u}, q_{d}\right), q_{u}=e$ and $q_{d}=-e$ and $\mathcal{A}_{\mu}=\delta_{\mu 2} x_{1} B$.

\subsection{Thermodynamic Potential}

The mean-field thermodynamic potential at zero temperature $T$ and finite chemical potential $\mu$ is given by ${ }^{3}$ :

$$
\Omega_{0}=\frac{\left(m-m_{c}\right)^{2}+\Delta^{2}}{4 G}-4 \sum_{s= \pm 1} \int \frac{d^{3} k}{(2 \pi)^{3}} \sqrt{\left(E_{k}+s \mu\right)^{2}+\Delta^{2}}
$$


with $E_{k}=\sqrt{k^{2}+m^{2}}$, with $m$ being the dressed quark mass. To include magnetic field the effects we follow Menezes et al. ${ }^{6}$, making the replacements

$$
2 \int \frac{d^{3} k}{(2 \pi)^{3}} \rightarrow \sum_{f=u}^{d} \frac{\left|q_{f}\right| B}{4 \pi} \sum_{l=0}^{\infty} \alpha_{l} \int_{-\infty}^{+\infty} \frac{d k_{3}}{2 \pi}, \quad E_{k} \rightarrow E_{k_{3}, l}=\sqrt{k_{3}^{2}+2 l\left|q_{f}\right| B+m^{2}}
$$

where $\alpha_{l}=2-\delta_{l, 0}$ takes into account the degeneracy of the Landau levels.

\section{Regularization Schemes and Parametrization}

The NJL model is non-renormalizable, therefore a proper regularization scheme is required to avoid ultraviolet divergences. In this work we use two different schemes; the first is based on form factor function, and implemented through the prescription

$$
\sum_{l=0}^{\infty} \int_{-\infty}^{\infty} \frac{d k_{3}}{2 \pi} \rightarrow \sum_{l=0}^{\infty} \int_{-\infty}^{\infty} \frac{d k_{3}}{2 \pi} U_{\Lambda}\left(\sqrt{k_{3}^{2}+2 l\left|q_{f}\right| B}\right)
$$

Functions of Wood-Saxon types have the form

$$
U_{\Lambda}^{W S \alpha}(x)=\left[1+e^{\frac{x / \Lambda-1}{\alpha}}\right]^{-1}
$$

with the usual value $\alpha=0.05$. In the other scheme, the Magnetic Field Independent Regularization (MFIR ${ }^{7}$, the magnetic contributions are completely separated from the divergent terms. In this case, the final form of the thermodynamic potential is:

$$
\Omega_{0}(m, \Delta, B, \mu)=\Omega_{0}+\Omega_{\mathrm{Mag}}+\Omega_{B, \mu},
$$

with

$$
\begin{gathered}
\Omega_{\mathrm{Mag}}=-\frac{N_{c}}{\pi^{2}}(e B)^{2}\left\{\zeta^{\prime}\left(-1, x_{f}\right)-\frac{1}{2}\left(x_{f}^{2}-x_{f}\right) \ln \left(x_{f}\right)+\frac{x_{f}^{2}}{4}\right\} \\
\Omega_{B, \mu}=\frac{N_{c}}{2 \pi^{2}}(e B) \int_{0}^{\infty} d k_{3}\left\{\sum_{l=0}^{\infty} \alpha_{l} F\left(k_{3}^{2}+2 l e B\right)-2 \int_{0}^{\infty} d y F\left(k_{3}^{2}+2 y e B\right)\right\}, \quad(5) \\
F\left(z^{2}\right)=\sum_{s= \pm 1}\left[\sqrt{\left(\sqrt{z^{2}+m^{2}}+s \mu\right)^{2}+\Delta^{2}}-\sqrt{z^{2}+m^{2}+\Delta^{2}}\right], \text { and } x_{f}=\frac{m^{2}+\Delta^{2}}{2 e B}
\end{gathered}
$$

$\Omega_{0}$ is given in Eq. (1).

In general, the model parameters $G, m_{c}$ and $\Lambda$ are fixed in terms of the empirical values of $m_{\pi}, f_{\pi}$ and $\langle q \bar{q}\rangle_{0}$. However, the known values of these quantities are valid for $N_{c}=3$ case. For two colors case we use a $N_{c}$ scaling of physical quantities ${ }^{8}$. Thereby we fix $f_{\pi}=75.45 \mathrm{MeV}, m_{\pi}=140 \mathrm{MeV}$ and $\langle q \bar{q}\rangle_{0}^{1 / 3}=-218 \mathrm{MeV}$ and obtain, for WS0.05 the values $m(0)=311.865 \mathrm{MeV}, m_{c}=5.401 \mathrm{MeV}, G=7.39$ $\mathrm{GeV}^{-2}$ and $\Lambda=650 \mathrm{MeV}$; and, for MFIR, the values $m(0)=305.385 \mathrm{MeV}, m_{c}=$ $5.400 \mathrm{MeV}, G=7.23 \mathrm{GeV}^{-2}$ and $\Lambda=657 \mathrm{MeV}$. 


\section{Numerical Results and Remarks}

At $\mu_{B}=0$, there is no condensate formation in the system, and $\Delta=0$, and when increasing the baryon chemical potential the system goes to BEC state, where $\Delta \neq 0$ through a second order phase transition ${ }^{4}$, for any value of $e B$. It is well-known that the pion mass $m_{\pi}$ is as a function of the magnetic field, $m_{\pi}(e B)^{9}$, and having in mind that, for $N_{c}=2$ it is possible to evaluate analytically that BEC phase transition happens at $\mu_{B}=m_{\pi}$ (see L. He et al., ${ }^{3}$ and references therein), we show that, even though $m_{\pi}$ is a function of the magnetic field, the phase transition will always happen at $\mu_{B_{c}}^{B E C}=m_{\pi}(e B)$, for a given value of $e B$. This is shown in Fig. 1(a). Further increasing the chemical potential the system undergoes a BECBCS crossover, controlled by the quantity $\mu_{N}=\mu-m$. A value of $\mu_{N}<0$ is typical of BEC state, while $\mu_{N}>0$ is typical of BCS state ${ }^{3}$. In Fig. 1(b) we may see $\mu_{N}$ as a function of $\mu_{B}$ for different values of $e B$. Once the BEC phase transition is of second order we may use the Ginzburg-Landau expansion ${ }^{10}$ to determinate the critical chemical potential $\mu_{B_{c}}^{B E C}$ in Fig. 1(c). Otherwise, $\mu_{B_{c}}^{B E C-B C S}$, is obtained by making $\mu_{N}=0$, as can be seen on Fig. $1(\mathrm{~d})$. It is possible to see that there is an inverse magnetic catalysis (IMC) for small values of $e B$, and a magnetic catalysis (MC) for large values of $e B$.
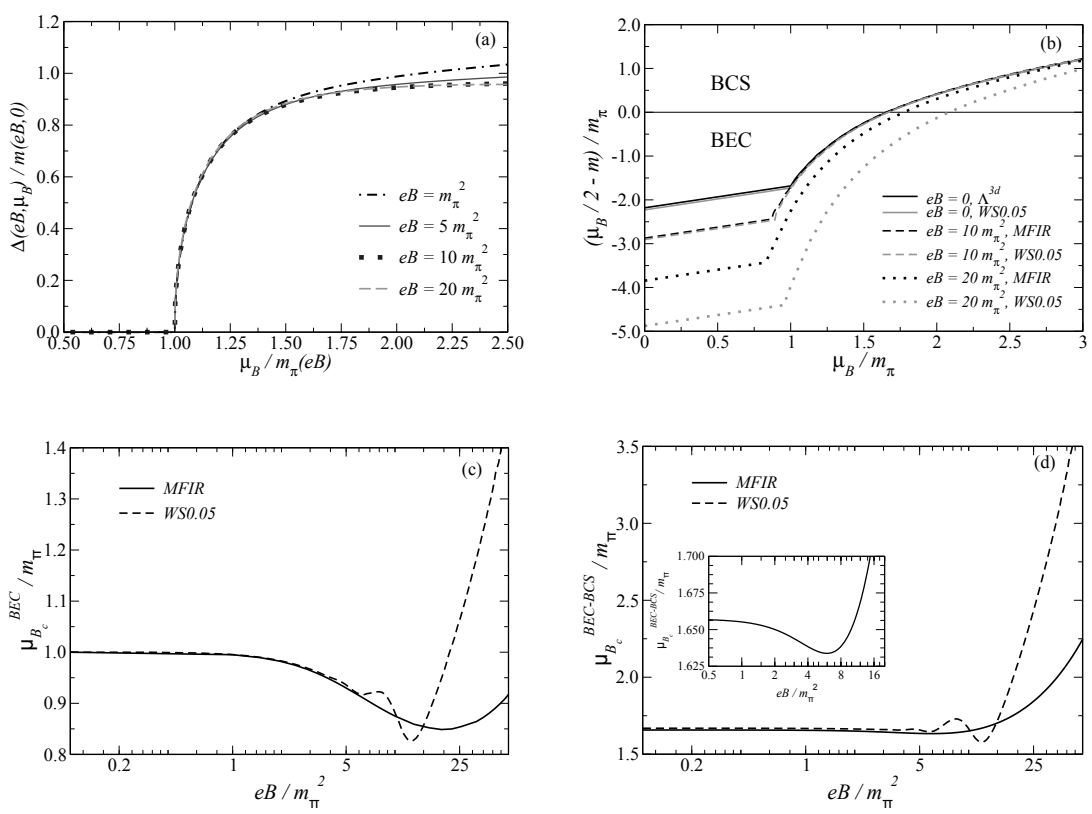

Fig. 1. (a) Order parameter $\Delta$ as a function of $\mu_{B}$ (normalized by the mass $m$ evaluated at $\mu=0$ and $e B \neq 0)$, showing that the phase transition is second order, and happens at $\mu_{B_{c}}^{B E C}=m_{\pi}(e B)$, for different values of $e B$. (b) Reference chemical potential $\mu_{N}$ as a function of $\mu_{B}$, for WS0.05 and MFIR and different values of $e B$. (c) $\mu_{B_{c}}^{B E C}$ and (d) $\mu_{B_{c}}^{B E C-B C S}$, as functions of the magnetic field, comparing the three regularization schemes. The inset plot on (d) shows a region with a weak IMC for MFIR. 
We recall that there are other regularization methods, like those based on form factor functions, that tend to produce nonphysical behaviors, like the presence of oscillations (unrelated to the van Alphen-de Haas oscillations) in the thermodynamic quantities. These artifacts of those regularization procedure are avoided in the MFIR scheme, which makes a full separation of the divergences from magnetic terms, that are all finite. For more details, please see Duarte et al. ${ }^{4}$.

\section{Acknowledgments}

Work partially supported by Capes, CNPq and FAPERJ (Brazilian agencies) and also CONICET and ANPCyT (Argentinian agencies).

\section{References}

1. F. Karsch, Lect. Notes Phys. 583, 209 (2002).

2. K. Fukushima, Journal of Physics G 39, 013101 (2012).

3. G. Sun, L. He, and P. Zhuang, Phys. Rev. D 75, 096004 (2007).

4. D. C. Duarte, P. G. Allen, R. L. S. Farias, P. H. A. Manso, R. O. Ramos, and N. N. Scoccola, Phys. Rev. D 93, 025017 (2016).

5. C. Ratti and W. Weise, Phys. Rev. D 70, 054013 (2004).

6. D. P. Menezes, M. B. Pinto, S. S. Avancini, A. P. Martínez, and C. Providência, Phys.Rev. C 79, 035807 (2009).

7. P. Allen, A. G. Grunfeld, and N. N. Scoccola, Phys. Rev. D 92, 074041 (2015).

8. T. Brauner, K. Fukushima, and Y. Hidaka, Phys. Rev. D 80, 074035 (2009).

9. S. S. Avancini, W. R. Tavares, and M. B. Pinto, Phys. Rev. D 93, 014010 (2016)

10. L. He, Phys. Rev. D 82, 096003 (2010). 